\title{
A COMPARATIVE STUDY ON ANTI-INFLAMMATORY DRUG COMBINATIONS IN DOMESTIC PIGEONS WITH EXPERIMENTALLY INDUCED ACUTE ARTHRITIS
}

\author{
Sajedeh SALANDARI ${ }^{1}$, Tahoora ShOMALI ${ }^{1 *}$, Najmeh MosleH $^{2}$ and Saeed NAZIFI ${ }^{2}$ \\ ${ }^{1}$ Division of Pharmacology and Toxicology, Department of Basic Sciences and \\ ${ }^{2}$ Department of Clinical Studies, School of Veterinary Medicine, Shiraz University, \\ P.O. Box 71441-69155, Shiraz, Iran
}

(Received 7 April 2019; accepted 27 August 2019)

\begin{abstract}
The study compares the effect of one-time administration of nonsteroidal and/or steroidal anti-inflammatory combinations by topical or intramuscular (IM) routes to pigeons with monosodium urate (MSU)-induced arthritis. Forty-five adult domestic pigeons were assigned into nine equal groups: $\mathrm{NC}$, negative control; PC, positive control with arthritis; sham, sham control; T1, meloxicam + hydrocortisone; T2, dexamethasone + piroxicam; T3, meloxicam + dexamethasone; T4, hydrocortisone + piroxicam; T5, dexamethasone + hydrocortisone; T6, meloxicam + piroxicam. Arthritis was also induced in T1 to T6 birds. Meloxicam and dexamethasone were administered by IM injection and the other drugs topically right after the induction of arthritis. Different drug combinations significantly decreased one-leg standing time. Induction of arthritis significantly increased TNF- $\alpha$ and IL-6 levels in synovial fluid and serum corticosterone and epinephrine in the PC group. Administration of drugs to birds of Groups T1 and T5 did not significantly change corticosterone concentration, while all different drug combinations decreased epinephrine level. Drug combinations that demonstrated better analgesic effect more strongly reduced serum epinephrine concentration. Meloxicam + hydrocortisone was the most effective combination in reducing inflammatory cytokines. In conclusion, one-time combination therapy with anti-inflammatory agents was effective in the acute management of inflammatory pain due to MSUinduced arthritis in pigeons, even by the topical route.
\end{abstract}

Key words: Anti-inflammatory drugs, arthritis, pain, cytokines, pigeon

Diverse groups of pathological conditions, from simple traumatic lesions to infectious diseases of inflammatory nature, are associated with both acute and chronic pain in avian species. Birds have developed peripheral and central pathways for the detection, transmission, modulation and perception of pain due to noxious stimuli (Machin, 2014) and therefore can experience the unpleasant feeling of pain that can negatively affect their behaviour, performance and even the

*Corresponding author; E-mail: tshomali@shirazu.ac.ir; Phone: 0098 (71) 3613-8907 
outcome of the therapeutic approach for the underlying disease. On the other hand, proper pain management in birds is mandatory for humane and ethical reasons.

Inflammatory pain is a commonly encountered clinical situation in avian species. Non-steroidal anti-inflammatory drugs (NSAIDs) are well known for their analgesic effect, which originates not only from their ability to peripherally reduce inflammation but also from their central anti-nociceptive properties (Riviere and Papich, 2009). Although the results of pain management with NSAIDs are not consistent in different avian species, NSAID administration has shown positive effects in this regard, and drugs like meloxicam and carprofen are commonly used for inflammatory pain management in avian species (Malik and Valentine, 2018).

Moreover, as one of the features in the long and diverse list of their effects on different body functions, glucocorticoids or steroidal anti-inflammatory drugs (SAIDs) can reduce pain intensity by inhibiting prostaglandin synthesis and reducing vascular permeability (Leppert and Buss, 2012); for instance, betamethasone has been shown to effectively reduce inflammatory joint pain of chickens (Hocking et al., 2001).

Currently, different agents from the NSAID or SAID classes are commercially available that may be used in avian medicine and are administered by different routes. Unfortunately, the lack of avian species-specific information on effective drugs and proper protocols for pain management (Mazor-Thomas et al., 2014), especially for combinatorial drug protocols, complicates the process of pharmaceutical pain management in clinical settings and makes it a hard task for the clinician to select the proper approach for pain management in these species. The situation is worsened further by the high sensitivity of ornamental and wild birds to stress, e.g. due to the administration of drugs by injection. This reduces the willingness of clinicians to administer dugs by this route in favour of other routes like oral administration of drugs usually in the feed or water. However, this decision can deprive the patient from some of the advantages of the IM route, such as administering a precise amount of drug in a short time to a bird which may not be eager to consume food and/or water due to the nature of the disease or its accompanying factors like pain. On the other hand, administration of drugs by the topical route, where applicable, is a relatively lower-stress procedure that can also reduce the chance for some of the drug-induced adverse effects. Therefore, acquiring knowledge about optimised protocols in order to gain more efficient and/or faster responses is greatly needed.

Since 1962 when Faires and McCarty provoked inflammatory reactions in the knee joint of dogs and men injected with monosodium urate (MSU) microcrystals, the inflammatory nature of this model has been frequently shown in other animals, especially rodents. For instance, inflammatory cell infiltration and

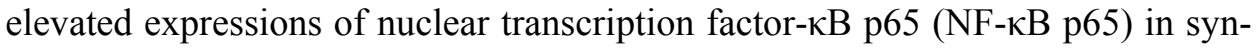


ovial tissues accompanied by increased levels of tumour necrosis factor- $\alpha$ (TNF$\alpha$ ) and different interleukins (ILs) in the synovial fluid or serum of rats and mice have been reported (Liu-Bryan et al., 2005; Kou et al., 2015; Dhanasekar and Rasool, 2016). Although MSU-induced arthritis in birds is also an inflammatory process, knowledge on the possible changes in inflammatory cytokines is scarce in avian species.

The microcrystal arthritis model for acute inflammatory pain is among the few pain models that have been successfully evaluated in pigeons. This model has the ability to reflect both nociception and inflammation, and can be properly used for the evaluation of pharmaceutical agents for pain control (Brune et al., 1974). In chickens, it has been shown that the pain due to microcrystalline MSU injection is associated with increased sensitivity of the joint capsule C-fibre receptors as the peripheral neural component for the pain (Gentle et al., 1999). Moreover, it has been clarified that pain due to acute inflammatory joint conditions is processed in specific areas of the parrot cerebrum, including the nidopallium (Paul-Murphy et al., 2005).

The effect of monotherapy with different analgesic agents has been evaluated in avian models with MSU-induced arthritis and has been associated with different outcomes. Some examples include subcutaneous colchicine in pigeons (Floersheim et al., 1973), bupivacaine (Hocking et al., 1997), morphine sulphate and fentanyl citrate (Gentle et al., 1999), betamethasone, dexamethasone (sodium phosphate) and methylprednisolone (Hocking et al., 2001) as well as NSAIDs (carprofen, flunixin, ketoprofen and sodium salicylate, intramuscularly) in fowls (Hocking et al., 2005) and meloxicam administered by IM injection in Hispaniolan parrots (Cole et al., 2009). As far as we know, there is no information on the possible outcome of one-time combination therapy with anti-inflammatory agents for the acute management of pain in this model.

This study compares the therapeutic outcome (pain-relieving effect determined by the time that the birds stand on the contralateral leg) of NSAID and/or SAID combinations that are administered by topical or IM routes to pigeons with experimentally induced arthritis. Selected biochemical parameters related to stress/pain (serum epinephrine and corticosterone levels) or inflammation (IL-6 and TNF- $\alpha$ levels in the synovial fluid) were also assayed.

\section{Materials and methods}

\section{Birds and study design}

Forty-five adult, clinically healthy domestic pigeons (Columba livia domestica) from both sexes with a body weight of $250 \pm 50 \mathrm{~g}$ that had been raised in the same aviary throughout their life were used in this study. The birds were transferred to isolated rooms with controlled temperature $\left(22^{\circ} \mathrm{C}\right)$ and a $10 / 14$ hours 
light/dark cycle. They had free access to a commercial pellets for pigeons (Tiba Bird $^{\circledR}$, Iran) and tap water and were kept in metal cages (five birds randomly allocated to each cage with at least $3,600 \mathrm{~cm}^{2}$ area considered for each bird) that were equipped with perches.

Table 1

Experimental design

\begin{tabular}{|c|c|c|c|c|c|c|c|}
\hline \multirow{3}{*}{ Group } & \multicolumn{7}{|c|}{ Treatments } \\
\hline & \multirow{2}{*}{ Arthritis } & \multicolumn{3}{|c|}{ Pectoral muscle injection } & \multicolumn{3}{|c|}{ Topical tarsus } \\
\hline & & Saline & $\begin{array}{c}\text { Dexa- } \\
\text { methasone }\end{array}$ & Meloxicam & Petrolatum & $\begin{array}{l}\text { Hydro- } \\
\text { cortisone }\end{array}$ & Piroxicam \\
\hline $\mathrm{NC}$ & - & - & - & - & - & - & - \\
\hline SHAM & - & + & - & - & + & - & - \\
\hline $\mathrm{PC}$ & + & + & - & - & + & - & - \\
\hline $\mathrm{T} 1$ & + & + & - & + & - & + & - \\
\hline $\mathrm{T} 2$ & + & + & + & - & - & - & + \\
\hline T3 & + & - & + & + & - & - & - \\
\hline $\mathrm{T} 4$ & + & + & - & - & - & + & + \\
\hline T5 & + & + & + & - & - & + & - \\
\hline T6 & + & + & - & + & - & - & + \\
\hline
\end{tabular}

NC: negative control; PC: positive control with arthritis; sham: sham control; T1: birds with arthritis that received injectable meloxicam + topical hydrocortisone; T2: birds with arthritis that received injectable dexamethasone + topical piroxicam; T3: birds with arthritis that received injectable meloxicam + injectable dexamethasone; T4: birds with arthritis that received topical hydrocortisone + topical piroxicam; T5: birds with arthritis that received injectable dexamethasone + topical hydrocortisone; T6: birds with arthritis that received injectable meloxicam + topical piroxicam

The acclimatisation period for birds was one week; each cage containing five birds was randomly assigned to treatment groups as follows (Table 1): (1) Negative control (NC) group: the birds did not receive any special treatment during the experiment; (2) Sham control (sham) group: the birds received $0.05 \mathrm{~mL}$ of normal saline by intra-articular injection into the left intertarsal joint, accompanied by two-sided IM injection of normal saline in the pectoral muscles and topical administration of petrolatum (Vaseline ${ }^{\circledR}$, USA) circumferentially on the left intertarsal joint; (3) Positive control (PC) group: acute inflammatory arthritis was induced by aseptically injecting $0.05 \mathrm{~mL}$ of autoclaved microcrystalline sodium urate (Sigma, USA) in normal saline ( $8 \%$ suspension) into the left intertarsal joint. The method for induction of arthritis in pigeons was adopted from Brune et al. (1974). These pigeons also received IM injections of normal saline and topically administered petrolatum as previously described for the sham group. Arthritis was also induced in the left intertarsal joint of all birds in Groups T1-T6 as stated above and then different therapeutic approaches were used in these groups as follows: (4) Group T1: meloxicam (IM) + topical hydrocortisone 
cream; (5) Group T2: dexamethasone (IM) + topical piroxicam; (6) Group T3: dexamethasone (IM) + meloxicam (IM); (7) Group T4: topical hydrocortisone + piroxicam; (8) Group T5: dexamethasone (IM) + topical hydrocortisone; (9) Group T6: meloxicam (IM) + topical piroxicam.

Injectable solutions (NSAID or SAID) were administered in the left pectoral muscle and normal saline in the right pectoral muscle of the birds. The volume of normal saline for IM injection was calculated as the volume of its counterpart drug in a pigeon with similar body weight. In Group T3 NSAID was injected in the left pectoral muscle and SAID in the right pectoral muscle. The birds received meloxicam (Meloxivet ${ }^{\circledR} 2 \%$ injectable solution in $50 \mathrm{~mL}$ vial, Razak Pharmaceutical Lab., Iran) at $0.5 \mathrm{mg} \mathrm{kg}^{-1}$ (Cole et al., 2009) and/or dexamethasone (Dexacoid ${ }^{\circledR} 0.4 \%$ injectable solution in 20-mL vial, Nasr Pharmaceutical Co., Iran) at $2 \mathrm{mg} \mathrm{kg}^{-1}$ (Plumb, 2008). Piroxicam (Piroxicam-Najo ${ }^{\circledR} 0.5 \%$ gel, Iran Najo Pharmaceutical Co., Iran) or hydrocortisone cream (Cortinil ${ }^{\circledR} 1 \%$, Sina Darou, Iran) was used topically on the left intertarsal joint as petrolatum administration.

Each pigeon was weighed on the day before starting the experiment and administration of drugs was performed immediately after injecting MSU for the induction of arthritis.

All procedures used in this study are in accordance with our institutional ethical guidelines which are based on the EU guidelines for animal experiments. The study design and procedures were approved by our institutional ethical committee before commencing the experiment.

\section{Recording of standing behaviour}

After intra-articular injection of MSU or normal saline, each bird was immediately transferred to a transparent glass cage and its behaviour was recorded by a camera fixed in a corner of the cage from 120-180 min post injection. The standing time on the right leg during this period was recorded. Each footage was checked twice by an observer unaware of the grouping system and the sum of the 'one-leg-only standing time' was determined for each bird. Choosing the time period was based on the study by Brune et al. (1974) demonstrating that pigeons show the most prominent one-leg standing behaviour during this period of time in an MSU-induced arthritis model.

\section{Blood sampling, epinephrine and corticosterone assays}

At the end of the recording time, blood samples (maximum $2.5 \mathrm{~mL}$ ) were obtained by venipuncture of the wing vein using a $2.5-\mathrm{mL}$ sterile syringe with $23 \mathrm{G}$ needle size and transferred to plain glass tubes without anticoagulant. Sampling of all birds was performed from 10 to 11 a.m. Blood samples were centrifuged at $2800 \mathrm{~g}$ for $5 \mathrm{~min}$ and sera were harvested and stored at $-20^{\circ} \mathrm{C}$ until analysis. 
Serum epinephrine level was determined by sandwich ELISA method using chicken epinephrine ELISA kit as described by the manufacturer (Bioassay Technology Lab., China). For determination of serum corticosterone concentration sandwich chicken corticosterone ELISA kit was used (Bioassay Technology Lab.). The intra-assay and inter-assay coefficients of variation (CVs) were $<8 \%$ and $<10 \%$, respectively, for both kits.

\section{Synovial lavage and determination of IL-6 and TNF- $\alpha$}

After blood sampling, birds were euthanised by decapitation, the left intertarsal joint was washed with $0.5 \mathrm{~mL}$ normal saline, and the synovial lavage fluid was collected and stored at $-20^{\circ} \mathrm{C}$.

Levels of TNF- $\alpha$ and IL-6 in the synovial fluid were assayed by sandwich ELISA kits for pigeon TNF- $\alpha$ and IL-6 (MyBioSource Co., USA). The intra- and inter-assay CVs were both $<15 \%$.

\section{Statistical analysis}

Data are presented as mean \pm standard deviation (SD). Data analysis was performed by one-way analysis of variance method followed by Tukey's multiple comparison test by using GraphPad Prism Version 6 (GraphPad Software Inc., USA).

\section{Results}

\section{One-leg standing time}

Birds in the PC group showed one-leg standing behaviour for a period of time that was significantly longer than for the NC birds $(\mathrm{P}<0.0001)$ (Fig. 1). Treating birds with all different drug combinations was associated with a significant decrease in one-leg standing time as compared to $\mathrm{PC}$ birds $(\mathrm{P}<0.0001$ for all comparisons). Except for birds in the T4 $(\mathrm{P}<0.0001)$ and T6 $(\mathrm{P}<0.01)$ groups that showed significantly longer one-leg standing behaviour as compared to NC birds, administration of drug combinations to other groups resulted in oneleg standing times that were not significantly different from that of the NC birds $(\mathrm{P}>0.05)$. Birds in Group T4 showed the longest one-leg standing time among the treated groups, which was significantly longer than for all other treated groups ( $\mathrm{P}<0.0001$ for all comparisons). The time of one-leg standing was not significantly different in groups $\mathrm{T} 1, \mathrm{~T} 2, \mathrm{~T} 3$ and $\mathrm{T} 5(\mathrm{P}>0.05)$.

\section{Level of TNF- $\alpha$ in the synovial fluid}

Induction of arthritis was associated with a significant increase in TNF- $\alpha$ level in the synovial fluid of birds in group $\mathrm{PC}$ as compared to NC pigeons $(\mathrm{P}<$ $0.0001)$. Among the treated groups (T1-T6), only birds in Group T1 showed a sig- 
nificantly reduced concentration of TNF- $\alpha$ as compared to PC birds $(\mathrm{P}<0.001)$. Interestingly, administration of injectable NSAID (meloxicam) + topical SAID (hydrocortisone) to these birds reversed TNF- $\alpha$ level to its concentration in the NC group $(\mathrm{P}>0.05)$. Birds in Groups T2 and T5 showed significantly higher levels of TNF- $\alpha$ compared to Group T1 ( $<0.05$ and $\mathrm{P}<0.001$, respectively) (Fig. 2).

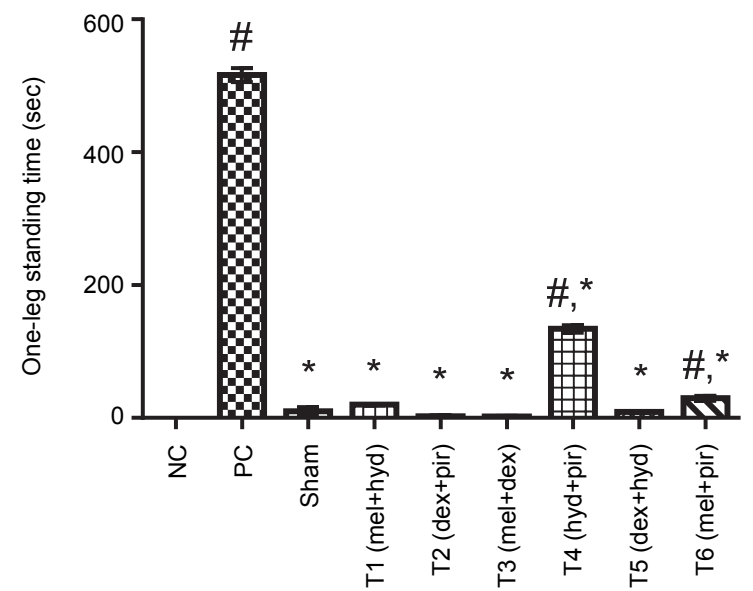

Fig. 1. Time of standing on one leg (mean $\pm \mathrm{SD}$ ) in birds of the different groups. NC: negative control, PC: positive control with arthritis, sham: sham control, T1: birds with arthritis that received injectable nonsteroidal anti-inflammatory drug (NSAID) + topical steroidal anti-inflammatory drug (SAID), T2: birds with arthritis that received injectable SAID + topical NSAID, T3: birds with arthritis that received injectable NSAID + injectable SAID, T4: birds with arthritis that received topical SAID + topical NSAID, T5: birds with arthritis that received injectable SAID + topical SAID, T6: birds with arthritis that received injectable NSAID + topical NSAID. Mel: meloxicam; Hyd: hydrocortisone; Dex: dexamethasone; Pir: piroxicam. ${ }^{\#}$ Significantly different from the NC group; ${ }^{*}$ significantly different from the PC group $(\mathrm{P}<0.05)$

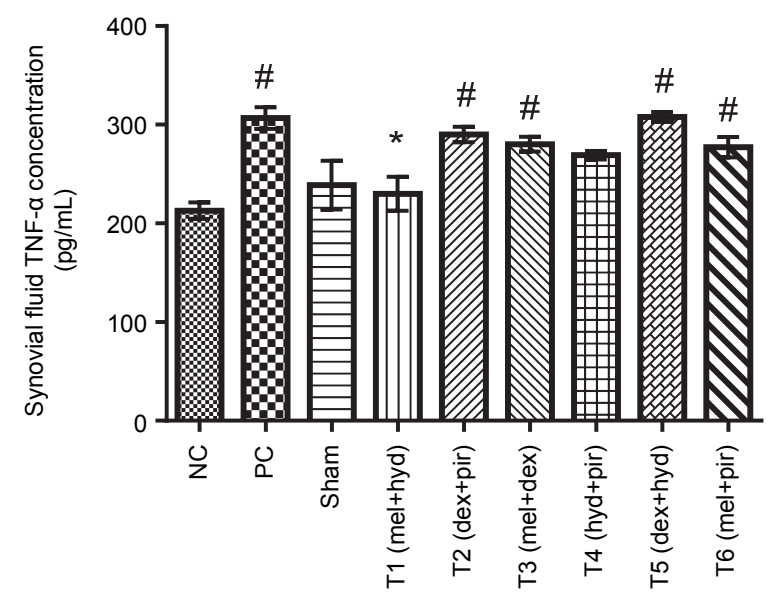

Fig. 2. Concentration of TNF- $\alpha$ (mean $\pm \mathrm{SD}$ ) in birds of the different groups (see legend to Fig. 1) ${ }^{\#}$ Significantly different from the NC group; ${ }^{*}$ significantly different from the $\mathrm{PC}$ group $(\mathrm{P}<0.05)$ 


\section{Level of IL-6 in the synovial fluid}

As shown in Fig. 3, induction of arthritis in birds of Group PC resulted in a significant increase in IL-6 concentration in their synovial fluid as compared to $\mathrm{NC}$ birds $(\mathrm{P}<0.05)$. Administration of different drug combinations to birds of Groups T1-T6 was not associated with a significant change in IL-6 concentration as compared to PC birds ( $\mathrm{P}>0.05)$, However, birds in Groups $\mathrm{T} 1$ and $\mathrm{T} 6$ showed the lowest concentration of IL-6 among the treated groups, which was statistically similar to that of the NC birds $(\mathrm{P}>0.05)$.

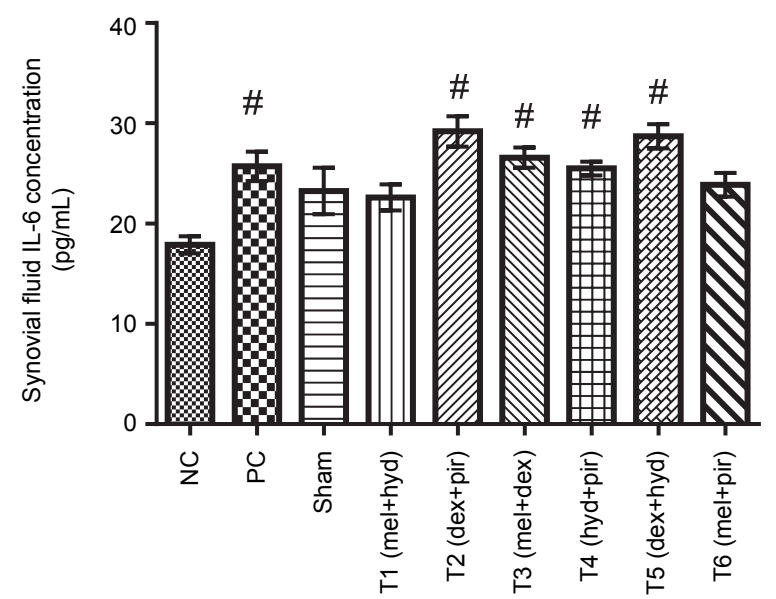

Fig. 3. Concentration of IL-6 (mean \pm SD) in birds of the different groups (see legend to Fig. 1).

${ }^{\#}$ Significantly different from the NC group; ${ }^{*}$ significantly different from the PC group $(\mathrm{P}<0.05)$

\section{Serum corticosterone concentrations}

Figure 4 shows that the induction of arthritis in birds of Group PC resulted in a significant increase in serum corticosterone level as compared to the $\mathrm{NC}$ group $(\mathrm{P}<0.0001)$. Sham birds showed significantly different levels of serum corticosterone as compared to both Groups PC and NC $(\mathrm{P}<0.0001)$. Administration of drugs to birds of Groups T1 and T5 was not associated with a significant change in serum corticosterone level as compared to Group PC $(\mathrm{P}>0.05)$. Other treated groups (T2, T3, T4 and T6) showed statistically similar concentrations of serum corticosterone $(\mathrm{P}>0.05)$ which were significantly lower than in Group PC ( $\mathrm{P}<0.01, \mathrm{P}<0.05, \mathrm{P}<0.01$ and $\mathrm{P}<0.0001$, respectively) and higher than in the $\mathrm{NC}$ group ( $\mathrm{P}<0.0001$ for all comparisons).

\section{Serum epinephrine concentrations}

Birds in the PC group showed a significantly increased level of serum epinephrine as compared to birds in the NC group $(\mathrm{P}<0.0001)$. All different drug 
combinations resulted in a significant decrease in serum epinephrine levels as compared to $\mathrm{PC}$ birds $(\mathrm{P}<0.0001$ for all comparisons); however, only the drug combinations that were administered to T2, T3 and T5 groups could reverse serum epinephrine level to that of the $\mathrm{NC}$ group $(\mathrm{P}>0.05)$. Serum epinephrine concentrations were statistically the same among these three (T2, T3 and T5) groups $(\mathrm{P}>0.05)$. The data are summarised in Fig. 5.

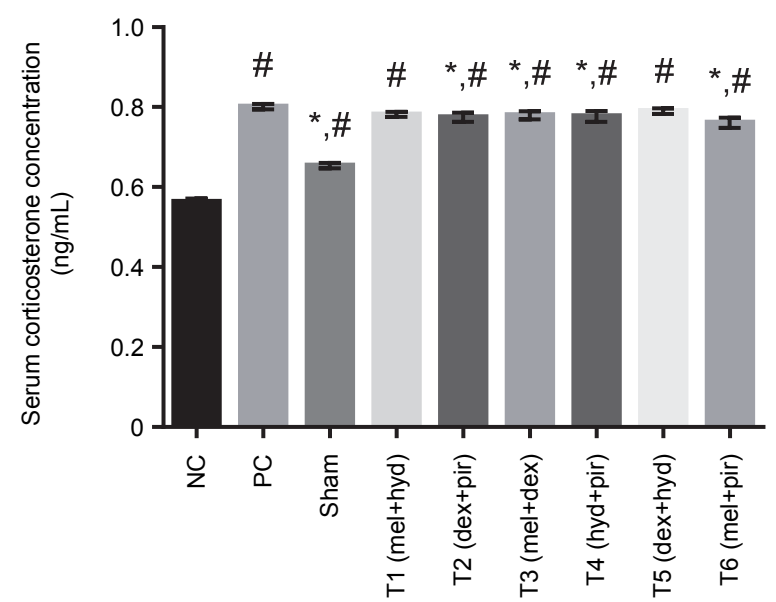

Fig. 4. Serum concentration of corticosterone (mean \pm SD) in birds of the different groups (see legend to Fig. 1). ${ }^{\#}$ Significantly different from the NC group; ${ }^{*}$ significantly different from the $\mathrm{PC}$ group $(\mathrm{P}<0.05)$

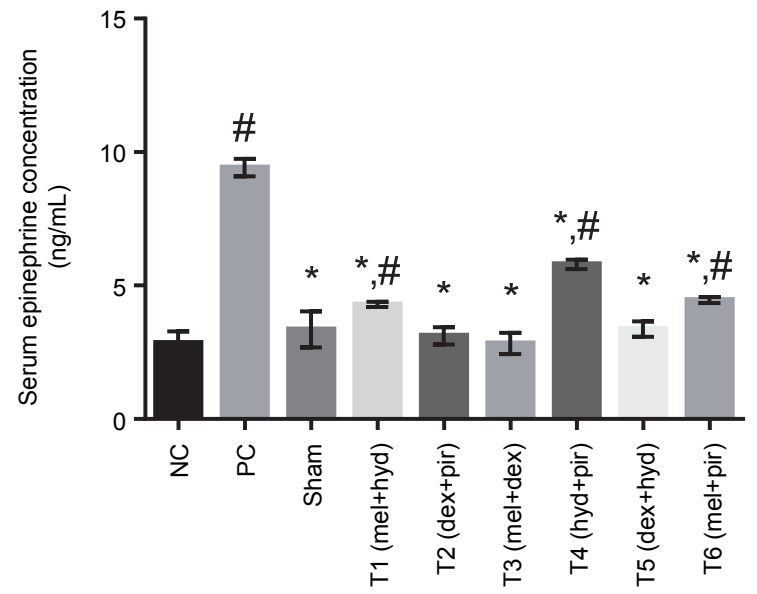

Fig. 5. Serum concentration of epinephrine $($ mean $\pm \mathrm{SD})$ in birds of the different groups (see legend to Fig. 1). ${ }^{\#}$ Significantly different from the NC group; ${ }^{*}$ significantly different from the $\mathrm{PC}$ group $(\mathrm{P}<0.05)$ 


\section{Discussion}

Anti-inflammatory drugs from both steroidal and nonsteroidal classes are common therapeutic agents that are successfully used to manage inflammatory pain in avian species. Corticosteroids usually show a very strong effect but their side effects limit their use, especially in long-term or high-dose regimens; moreover, some of their effects are elicited only after a relatively long time (Riviere and Papich, 2009) which is definitely not desired in acute conditions when there is a need for fast response.

In this study, the effects of one-time administration of different antiinflammatory drug combinations were evaluated by two routes (IM and/or topical) in pigeons with acute inflammatory pain. Choosing the drugs was based on the pathophysiologic process of the pain in this model.

The results indicate that the administration of a drug combination by the topical route (Group T4) improves the therapeutic outcome as shown by reduced one-leg standing time, although the effect was lower than for other drug combinations/routes. When one of the drugs was used by injection, administration of meloxicam + piroxicam showed lower effect than the combinations that included at least one steroidal agent, although the difference was significant only with the dexamethasone + piroxicam group. Interestingly, when both classes of drugs were administered by injection, the result was not statistically better than when only one drug was used by injection (except for the combination of two NSAIDs). Therefore, administration of the topical + injectable combination that includes at least one SAID is recommended.

Another interesting finding of the present study was the significant increase in synovial fluid TNF- $\alpha$ and IL- 6 concentrations in birds of the PC group that confirms the inflammatory nature of this model in pigeons. It has been shown that MSU crystals can induce IL-6 production in synoviocytes and monocytes, and that synovial fluid from patients with gout and pseudogout contains high levels of IL-6 (Guerne et al., 1989). Moreover, an association is observed between serum levels of IL- 6 and the presence of tophi and deformities in human patients with gout (Cavalcanti et al., 2016). TNF- $\alpha$ has a pivotal role in both acute and chronic inflammatory responses including rheumatoid arthritis. Blocking this cytokine has been associated with promising results in human patients and it is successfully targeted to treat rheumatoid arthritis in humans (Feldmann, 2002; di Comite et al., 2006). Recently, Rohde et al. (2018) have provided strong evidence for the existence of a fully functional TNF- $\alpha / \mathrm{TNF}-\alpha$ receptor system in birds with an ability to induce NF- $\mathrm{KB}$ expression. Although the role of this cytokine in MSU-induced arthritis models has been confirmed in mammals (LiuBryan et al., 2005; Kou et al., 2015; Dhanasekar and Rasool, 2016), to the best of our knowledge this is the first time that its involvement in an avian model of this type of arthritis has been demonstrated. As previously stated, among different 
drug combinations, the administration of meloxicam (intramuscularly) + hydrocortisone (topically) showed better results (especially on TNF- $\alpha$ levels) followed by Groups T4 and T6. It has been clearly demonstrated that glucocorticoids suppress TNF- $\alpha$ expression by diminishing the nuclear binding activity of transcription factors prior to and during the critical phase of TNF- $\alpha$ transcription (Steer et al., 2000). Two different glucocorticoids were used in this study including topical hydrocortisone, which is a low-potency glucocorticoid, and injectable dexamethasone, which is a high-potency glucocorticoid. Surprisingly, it was observed that the administration of hydrocortisone + meloxicam has better effect on IL-6 and TNF- $\alpha$ level than that of dexamethasone + meloxicam (although the difference was not statistically significant). This may be related to the fact that hydrocortisone was administered just at the site of inflammation and may have reached high concentrations in the synovium and synovial fluid, although this needs to be confirmed. On the other hand, topical administration of drugs close to their target site can limit their adverse effects; therefore, it seems that topical hydrocortisone may be a logical choice in inflammatory arthritis in pigeons.

Severe pain can profoundly affect the endocrine system with the hypothalamic-pituitary-adrenal axis influenced prior to others, with a resultant increase in serum glucocorticoid level (Tennant, 2013). Corticosterone is the major glucocorticoid hormone in birds that acts as a stress response constituent. This hormone can help birds confront with stressors by its metabolic actions such as increasing blood glucose levels (Cockrem et al., 2009). As it was expected in this study, induction of MSU arthritis in birds of the PC group was associated with a significant increase in serum corticosterone levels. Despite proper effects on oneleg standing behaviour, two of the drug combinations that contained hydrocortisone and an injectable agent (dexamethasone or meloxicam) were not successful in reducing the corticosterone levels as compared to the PC group, while when hydrocortisone was administered with piroxicam, the corticosterone level decreased significantly. Apart from the nature and effect of the concomitant drug, it seems that injection itself may be important as a stress factor. Consistently, levels of corticosterone in sham birds (without arthritic pain) also increased, which shows the stress imposed to the bird by the injection itself which, in turn, can complicate our results.

Like corticosterone, catecholamines have been used as a humoral index of stress in birds (Le Maho et al., 1992) and it has been shown that pain can stimulate the release of adrenalin and other catecholamines from the adrenal medulla (Tennant, 2013). Consistently, an appreciable increase in serum epinephrine concentration of birds in the PC group with MSU-induced arthritis was observed as compared to the NC group. On the other hand, sham birds showed statistically similar levels of epinephrine as compared to NC birds. Therefore, the injection stress was not reflected by this parameter. Regarding the effects of the drugs on this parameter, three drug combinations that contained dexamethasone (Groups 
T2, T3 and T5) showed the most profound decreasing effects on serum epinephrine levels. In a study on mice, it was observed that after stress, adrenocorticotropic hormone levels are elevated, and exogenous dexamethasone suppresses the production of endogenous corticosterone and phenylethanolamine N-methyltransferase (PNMT), the enzyme that converts norepinephrine to epinephrine in the adrenal medulla (Sharara-Chami et al., 2010). In the present study, although the level of serum corticosterone in Groups T2 and T3 decreased significantly as compared to PC birds, a significant reduction in serum corticosterone levels of Group T5 was not observed. It is worth mentioning that these three groups also had the best effect on reducing pain as shown by one-leg standing time. Therefore, it is more plausible that the analgesic effect of these drug combinations has led to the reduction of serum epinephrine levels.

One of the limitations of this study is that only the acute effects of drug combinations were evaluated in a short-term study, where MSU-induced arthritis was used as a model of acute inflammatory pain. Although no clinical adversities due to drug administration were found, this result does not exclude the adverse effects of these drugs, which are especially important in repetitive long-term administration protocols.

In conclusion, one-time combination therapy with NSAIDs and/or SAIDs was effective in the acute management of inflammatory pain due to MSUinduced arthritis in pigeons, even when both drugs were used topically. Drug combinations that demonstrated better analgesic effect (all included injectable dexamethasone) had a stronger reducing effect on serum epinephrine concentration. Injectable meloxicam + topical hydrocortisone was the most effective combination in reducing the levels of inflammatory cytokines, especially TNF- $\alpha$, in the synovial fluid.

\section{Acknowledgement}

Funding for this study was provided by Shiraz University, School of Veterinary Medicine (grant number: 96GCU3M163773).

\section{References}

Brune, K., Walz, D. and Bucher, K. E. (1974): The avian microcrystal arthritis I. Simultaneous recording of nociception and temperature effect in the inflamed joint. Agents Actions 4, 21-26.

Cavalcanti, N. G., Marques, C. D., Lins e Lins, T. U., Pereira, M. C., Rêgo, M. J., Duarte, A. L., Pitta, I. R. and Pitta, M. G. (2016): Cytokine profile in gout: inflammation driven by IL-6 and IL-18? Immunol. Invest. 45, 383-395.

Cockrem, J. F., Barrett, D. P., Candy, E. J. and Potter, M. A. (2009): Corticosterone responses in birds: individual variation and repeatability in Adelie penguins (Pygoscelis adeliae) and other species, and the use of power analysis to determine sample sizes. Gen. Comp. Endocrinol. 163, 158-168. 
Cole, G. A., Paul-Murphy, J., Krugner-Higby, L., Klauer, J. M., Medlin, S. E., Keuler, N. S. and Sladky, K. K. (2009): Analgesic effects of IM administration of meloxicam in Hispaniolan parrots (Amazona ventralis) with experimentally induced arthritis. Am. J. Vet. Res. 70, $1471-1476$.

Dhanasekar, C. and Rasool, M. (2016): Morin, a dietary bioflavonol suppresses monosodium urate crystal-induced inflammation in an animal model of acute gouty arthritis with reference to NLRP3 inflammasome, hypo-xanthine phospho-ribosyl transferase, and inflammatory mediators. Eur. J. Pharmacol. 786, 116-127.

di Comite, G., Marinosci, A., Di Matteo, P., Manfredi, A., Rovere-Querini, P., Baldissera, E., Aiello, P., Corti, A. and Sabbadini, M. G. (2006): Neuroendocrine modulation induced by selective blockade of TNF-alpha in rheumatoid arthritis. Ann. N. Y. Acad. Sci. 1069, 428-437.

Faires, J. S. and McCarty, D. J. (1962): Acute arthritis in man and dog after intrasynovial injection of sodium urate crystals. The Lancet 280, 682-685.

Feldmann, M. (2002): Development of anti-TNF therapy for rheumatoid arthritis. Nat. Rev. Immunol. 2, 364-371.

Floersheim, G. L., Brune, K. and Seiler, K. (1973): Colchicine in avian sodium urate and calcium pyrophosphate microcrystal arthritis. Agents Actions 3, 20-23.

Gentle, M. J., Hocking, P. M., Bernard, R. and Dunn, L. N. (1999): Evaluation of intraarticular opioid analgesia for the relief of articular pain in the domestic fowl. Pharmacol. Biochem. Behav. 63, 339-343.

Guerne, P. A., Terkeltaub, R., Zuraw, B. and Lotz, M. (1989): Inflammatory microcrystals stimulate interleukin-6 production and secretion by human monocytes and synoviocytes. Arthritis Rheum. 32, 1443-1452.

Hocking, P. M., Gentle, M. J., Bernard, R. and Dunn, L. N. (1997): Evaluation of a protocol for determining the effectiveness of pretreatment with local analgesics for reducing experimentally induced articular pain in domestic fowl. Res. Vet. Sci. 63, 263-267.

Hocking, P. M., Robertson, G. W. and Gentle, M. J. (2001): Effects of anti-inflammatory steroid drugs on pain coping behaviours in a model of articular pain in the domestic fowl. Res. Vet. Sci. 71, 161-166.

Hocking, P. M., Robertson, G. W. and Gentle, M. J. (2005): Effects of non-steroidal antiinflammatory drugs on pain-related behaviour in a model of articular pain in the domestic fowl. Res. Vet. Sci. 78, 69-75.

Kou, Y. Y., Li, Y. F., Xu, M., Li, W. Y., Yang, M. and Li, R. L. (2015): Effects of RuPeng15 Powder (RPP15) on monosodium urate crystal-induced gouty arthritis in rats. Evid. Based Complement. Alternat. Med. doi:10.1155/2015/527019

Le Maho, Y., Karmann, H., Briot, D., Handrich, Y., Robin, J. P., Mioskowski, E., Cherel, Y. and Farni, J. (1992): Stress in birds due to routine handling and a technique to avoid it. Am. J. Physiol. 263, R775-781.

Leppert, W. and Buss, T. (2012): The role of corticosteroids in the treatment of pain in cancer patients. Curr. Pain Headache Rep. 16, 307-313.

Liu-Bryan, R., Scott, P., Sydlaske, A., Rose, D. M. and Terkeltaub, R. (2005): Innate immunity conferred by toll-like receptors 2 and 4 and myeloid differentiation factor 88 expression is pivotal to monosodium urate monohydrate crystal-induced inflammation. Arthritis Rheum. 52, 2936-2946.

Machin, K. L. (2014): Recognition and treatment of pain in birds. In: Egger, C. M., Love, L. and Doherty, T. (eds) Pain Management in Veterinary Practice. First edition. John Wiley \& Sons, Inc. pp. 407-415.

Malik, A. and Valentine, A. (2018): Pain in birds: a review for veterinary nurses. Vet. Nursing J. 33, 11-25.

Mazor-Thomas, J. E., Mann, P. E., Karas, A. Z. and Tseng, F. (2014): Pain-suppressed behaviors in the red-tailed hawk (Buteo jamaicensis). Appl. Anim. Behav. Sci. 152, 83-91. 
Paul-Murphy, J., Sladky, K. K., McCutcheon, R. A., Steege, E. and Converse, A. K. (2005): Using positron emission tomography imaging of the parrot brain to study response to clinical pain. Paper presented at the 2005 Annual Conference of the Academy of Molecular Imaging. Orlando (FL), March 18-23, 2005.

Plumb, D. C. (2008): Plumb's Veterinary Drug Handbook, 6th edition. Blackwell Publishing, USA.

Riviere, J. E. and Papich, M. G. (2009): Veterinary Pharmacology and Therapeutics. 9th edition. Wiley-Blackwell, USA.

Rohde, F., Schusser, B., Hron, T., Farkašová, H., Plachy, J., Härtle, S., Hejnar, J., Elleder, D. and Kaspers, B. (2018): Characterization of chicken tumor necrosis factor- $\alpha$, a long missed cytokine in birds. Front. Immunol. 9, 605. doi:10.3389/fimmu.2018.00605

Sharara-Chami, R. I., Joachim, M., Pacak, K. and Majzoub, J. A. (2010): Glucocorticoid treatment - effect on adrenal medullary catecholamine production. Shock 33, 213-217.

Steer, J. H., Kroeger, K. M., Abraham, L. J. and Joyce, D. A. (2000): Glucocorticoids suppress tumor necrosis factor-alpha expression by human monocytic THP-1 cells by suppressing transactivation through adjacent NF-kappa B and c-Jun-activating transcription factor-2 binding sites in the promoter. J. Biol. Chem. 275, 18432-18440.

Tennant, F. (2013): The physiologic effects of pain on the endocrine system. Pain Ther. 2, 75-86. 